\title{
Effects of different nitrogen fertilizer levels on yield, leave color, and content of bioactive compounds of Pouzolzia zeylanica L. Benn
}

\author{
Tuyen T. X. Vo ${ }^{1 *}$, Tan D. Nguyen ${ }^{1}, \&$ Thuy M. Nguyen ${ }^{2}$ \\ ${ }^{1}$ Faculty of Agriculture and Natural Resources, An Giang University, An Giang, Vietnam \\ ${ }^{1}$ College of Agriculture, Can Tho University, Can Tho, Vietnam
}

\begin{abstract}
ARTICLE INFO
Research Paper

Received: November 10, 2018

Revised: January 05, 2019

Accepted: February 18, 2019

Keywords

Bioactive compounds

Color of leaves

Nitrogen fertilizer level

Pouzolzia zeylanica plant

${ }^{*}$ Corresponding author

Vo Thi Xuan Tuyen

Email: vtxtuyen@agu.edu.vn
\end{abstract}

\section{ABSTRACT}

The study was carried out to investigate the effect of 4 levels of nitrogen fertilizer $\left(10,15,20\right.$ and $25 \mathrm{~kg}$ urea $\left./ 1000 \mathrm{~m}^{2}\right)$ applied on the color of leaves (chlorophyll index SPAD and the red-purple color by a* value in Hunter' color system), yield and bioactive compounds content (anthocyanin, flavonoid, polyphenol and tannin) of Pouzolzia zeylanica plant. The results showed that different nitrogen fertilizer levels had significant effects $(P \leq 0.05)$ on leave color, yield and content of bioactive compounds of this plant. At the level of $20 \mathrm{~kg}$ urea $/ 1000 \mathrm{~m}^{2}$, the leaves had red-purple color and the obtained $\mathrm{a}^{*}$ value was 8.06 ; the index of chlorophyll SPAD was 38.36 ; the yield was $1.64 \mathrm{ton} / 1000 \mathrm{~m}^{2}$, and the content of bioactive compounds was higher than other nitrogen fertilizer levels. The contents of anthocyanin, flavonoid, polyphenol and tannin were $0.296 \mathrm{mg} \mathrm{CE} / \mathrm{g}$ dried material (DM), $5.03 \mathrm{mg}$ QE/g DM, $15.54 \mathrm{mg} \mathrm{GAE} / \mathrm{g}$ DM and 10.05 mg TAE/g DM, respectively.

Cited as: Vo, T. T. X., Nguyen, T. D., \& Nguyen, T. M. (2019). Effects of different nitrogen fertilizer levels on yield, leave color, and content of bioactive compounds of Pouzolzia zeylanica L. Benn. The Journal of Agriculture and Development 18(4), 10-18. 


\title{
Ảnh hưởng của mức bón phân đạm lên năng suất, màu sắc lá và hàm lượng các hợp chất có hoạt tính sinh học của cây thuốc dòi (Pouzolzia zeylanica L. Benn)
}

\author{
Võ Thị Xuân Tuyền ${ }^{1 *}$, Nguyễn Duy Tân ${ }^{1} \&$ Nguyễn Minh Thủy ${ }^{2}$ \\ ${ }^{1}$ Khoa Nông Nghiệp và Tài Nguyên Thiên Nhiên, Trường Đại Học An Giang, An Giang \\ ${ }^{2}$ Khoa Nông Nghiệp, Trường Đại Học Cần Thơ, Cần Thơ
}

THÔNG TIN BÀI BÁO
Bài báo khoa học
Ngày nhận: 10/11/2018
Ngày chỉnh sửa: 05/01/2019
Ngày chấp nhận: 18/02/2019
Từ khóa
Các hợp chất có hoạt tính sinh học
Cây thuốc dòi
Màu sắc lá
Mức độ bón phân đạm
*Tác giả liên hệ
Võ Thị Xuân Tuyền
Email: vtxtuyen@agu.edu.vn

\section{TÓM TẮT}

Nghiên cứu được thực hiện nhằm khảo sát ảnh hưởng của 4 mức độ bón phân đạm $(10,15,20$ và $25 \mathrm{~kg}$ urea/1000 m²) lên màu sắc lá (chỉ số diệp lục tố SPAD và giá trị $\mathrm{a}^{*}$ trong hệ màu Hunter), năng suất và hàm lượng các hợp chất sinh học (anthocyanin, flavonoid, polyphenol và tannin) của cây thuốc dòi. Kết quả cho thấy khi thay đổi các mức độ bón phân đạm khác nhau có ảnh hưởng rõ rệt lên màu sắc lá, năng suất và hàm lượng các hợp chất có hoạt tính sinh học của cây thuốc dòi $(P \leq 0,05)$. Ở mức đạm bón $20 \mathrm{~kg}$ urea/1000 $\mathrm{m}^{2}$, lá có màu tím đỏ và giá trị a* đo được là 8,06 ; chỉ số diệp lục tố SPAD là 38,36 ; năng suất 1,64 tấn/1000 $\mathrm{m}^{2}$ và hàm lượng các hợp chất có hoạt tính sinh học đều cao hơn so với các mức đạm khác. Hàm lượng anthocyanin, flavonoid, polyphenol và tannin lần lượt là $0,296 \mathrm{mg} \mathrm{CE} / \mathrm{g}$ nguyên liệu khô $(\mathrm{DM}) ; 5,03 \mathrm{mg}$ QE/g DM; 15,54 mg GAE/g DM và 10,05 mg TAE/g DM.

\section{1. Đặt Vấn Đề}

Cây thuốc dòi có tên khoa học Pouzolzia zeylanica L. Benn, thuộc họ Gai (Urticaceae), phát triển tốt ở điều kiện khí hậu Việt Nam và được trồng phổ biến ở khu vực Đồng bằng sông Cửu Long. Theo Đông y, cây thuốc dòi có vị ngọt nhạt, tính mát, có tác dụng chỉ khái, tiêu đờm, dùng chữa ho lâu ngày, ho lao, viêm họng và viêm thanh phế quản. Bên cạnh đó, cây thuốc dòi có chứa nhiều hợp chất có hoạt tính sinh học cao như isoflavone, alkaloid, polyphenol, tannin, flavonoid và glycoside. Những chất này có khả năng chống oxy hóa, kháng khuẩn, kháng nấm, ức chế sự phát triển của tế bào và ngăn ngừa ung thư (Le, 2007; Paul \& Saha, 2012; Vo, 2012).

Ở khu vực Đồng bằng sông Cửu Long, cây thuốc dòi được người dân sử dụng như một loại rau để ăn sống, nấu canh hoặc phối hợp với các loại nguyên liệu khác như mã đề, rễ tranh, lá dứa, mía lau... nấu nước uống để bồi dưỡng cơ thề, thanh nhiệt và trị ho. Trong những năm gần đây, các nhà khoa học không những quan tâm đặc biệt về thực vật làm thuốc mà còn nghiên cứu chiết tách và thử nghiệm tác dụng trị bệnh của chúng để tìm kiếm những hợp chất mới thay thế những chất hóa học mà có thể hạn chế được những tác dụng phụ trong điều trị bệnh. Nhiều công ty và doanh nghiệp không ngừng nghiên cứu và đẩy mạnh việc ứng dụng cây thuốc vào sản xuất thực phẩm chức năng và dược phẩm.

Tuy nhiên, việc nghiên cứu trên cây thuốc dòi hiện nay còn rất ít. Đặc biệt là kỹ thuật canh tác hầu như chưa được nghiên cứu. Phần lớn cây thuốc dòi được trồng xen canh trong các vườn cây ăn trái với diện tích nhỏ và chỉ xem là cây trồng phụ. Theo Chu \& ctv. (2006), cây thuốc cần nhiều chất dinh dưỡng để sinh trưởng, phát triển, ra hoa và làm củ. Cung cấp nitơ có ảnh hưởng lớn đến bộ máy quang hợp như sự phát triển của lá, diện tích lá, quá trình sinh tổng hợp protein và chlorophyll và ảnh hưởng đến quá trình quang hợp của thực 
vật (Bojović \& Marković, 2009). Phân đạm là một trong những loại phân thiết yếu giúp cho cây tăng trưởng nhanh, gia tăng diện tích lá. Nếu bón thiếu đạm cây sẽ cằn cõi, lá vàng, hoa không trổ hoặc thưa, ngược lại bón đạm nhiều cây mọc vóng, hoa quả ít, củ ít tinh bột, khó chế biến và phẩm chất kém. Tất cả các chất dùng để bón đều cần được áp dụng một cách tiết kiệm theo đúng nhu cầu cụ thể của loài cây thuốc và khả năng cung cấp của đất (WHO, 2003). Do đó, để đáp ứng được cho ngành công nghiệp sản xuất thuốc thì cần phải có những nghiên cứu về quy trình kỹ thuật canh tác, trong đó chế độ bón phân cũng là một trong những nhân tố có ảnh hưởng rất lớn đến năng suất, màu sắc lá và sự tổng hợp các chất có hoạt tính sinh học trong cây thuốc dòi.

\section{Vật Liệu và Phương Pháp Nghiên Cứu}

\subsection{Vật liệu nghiên cứu}

Nghiên cứu được thực hiện trên cây thuốc dòi thân tím đỏ (Pouzolzia zeylanica L. Benn). Nguồn giống hom được lấy từ một hộ nông dân trồng cây thuốc dòi tại xã Hòa Bình, huyện Chợ Mới, tỉnh An Giang. Phân đạm sử dụng là phân đạm Phú Mỹ.

Thiết bị sử dụng: Máy đo diệp lục tố (SPAD 502, Nhật); máy đo màu (Chroma Meter CR-400, Nhật); máy nghiền dược liệu (Polymix 90D, Thụy sĩ); máy đo hấp thu quang phổ (SP-1920, Nhật); bể điều nhiệt (Memmert, Pháp), Vortex.

Hóa chất: Thuốc thử Folin-cioalteau, Folindenis và các chất chuẩn gallic acid, quercetin, tannic acid được mua từ công ty hóa chất Sigma (St. Louis, Mo. USA). Aluminum chloride, sodium carbonate, sodium acetate, glacial acetic acid, potassium chloride, hydrochloric acid and ethanol được mua từ công ty Analytical Reagent (Xilong Chemical Co. Ltd., China).

\subsection{Phương pháp nghiên cứu}

\subsubsection{Bố trí thí nghiệm}

Thí nghiệm được bố trí tại khu Thực nghiệm Trường Đại học An Giang. Thời gian thực hiện từ tháng 8 - 10/2016. Thí nghiệm được bố trí theo kiểu khối hoàn toàn ngẫu nhiên với một nhân tố và 3 lần lặp lại. Nhân tố là mức độ bón phân đạm được khảo sát ở $(10,15,20,25 \mathrm{~kg}$ urea/1000 m²), tương ứng với các mức đạm bón lần lượt là 60 , 90, 120 và $150 \mathrm{~g}$ trên diện tích lô $6 \mathrm{~m} 2$ cho mỗi nghiệm thức (Hình 1).
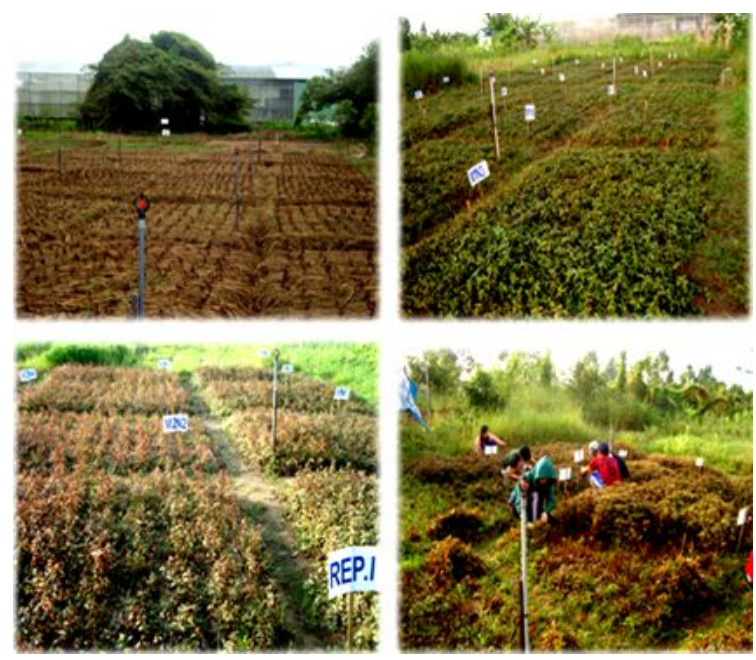

Hình 1. Một số hình ảnh trong bố trí thí nghiệm, trồng và thu hoạch cây thuốc dòi.

\subsubsection{Kỹ thuật trồng và chăm sóc}

Cách chọn hom: Chủ yếu lấy hom từ ngọn không quá non hoặc quá già nhằm giúp hom nhanh ra rễ và sinh trưởng khỏe. Chiều dài hom trung bình khoảng $10-15 \mathrm{~cm}$.

Cách trồng: Sử dụng 3 hom/hốc, sau 5 - 7 ngày sau khi trồng (NSKT) tiến hành dặm lại những chỗ hom mọc không đều để đảm bảo đúng mật độ trồng $(20 \times 20 \mathrm{~cm})$.

Bón phân: Đầu vụ trồng bón lót phân Super lân $\left(30 \mathrm{~kg} / 1000 \mathrm{~m}^{2}\right)$, phân kali $\left(30 \mathrm{~kg} / 1000 \mathrm{~m}^{2}\right)$, phân chuồng (phân bò) 2 tấn/1000 m². Đối với phân urea chia làm 3 lần bón ở 15,30 và 45 ngày sau khi trồng với tỷ lệ bón là $1 / 3$ trên tổng lượng phân cần bón.

Cách thu hoạch và tiêu chuẩn thu hoạch: Dùng dao hoặc kéo bén để thu hoạch cây thuốc dòi, cắt chừa phần gốc còn lại khoảng $5 \mathrm{~cm}$. Thu hoạch khi cây có chiều cao $25-30 \mathrm{~cm}$ (thời gian sinh trưởng 2 tháng sau khi trồng).

\subsubsection{Phân tích các chỉ tiêu hóa lý}

Phân tích đất trước khi trồng: Phân tích hàm lượng đạm tổng số theo phương pháp Micro Kjeldahl; lân tổng số theo phương pháp Loren; chất hữu cơ theo phương pháp Walkley Black, kali trao đổi và $\mathrm{pH}$ nước của đất (Truong, 1994; Do, 1999; Ngo, 2005). Cách lấy mẫu đất: lấy ở 5 
điểm ngẫu nhiên cho mỗi lần lặp lại sau đó trộn lại thành một mẫu. Số mẫu đất phần tích 3 mẫu.

Đo màu sắc của lá: Giá trị màu a* bằng máy (Chroma Meter CR-400, Nhật), chỉ số diệp lục tố bằng máy (Konica Minolta SPAD 502, Nhật). Đo vị trí lá thứ 3 và ở giữa lá trên 10 cây khác nhau cho mỗi mẫu thí nghiệm.

Năng suất thực tế: Thu hoạch toàn cây thuốc dòi trong lô thí nghiệm, cân khối lượng tươi và sau đó qui ra trên $1000 \mathrm{~m}^{2}$.

Trọng lượng tươi: Thu 5 bụi cho một ô thí nghiệm theo hình chéo góc, cân trọng lượng tươi lúc thu hoạch.

Trọng lượng khô: Cho các mẫu cây tươi vào túi giấy đem sấy ở $60^{\circ} \mathrm{C}$ đến khi mẫu khô hoàn toàn rồi đem mẫu cân (trừ túi giấy) thu được trọng lượng khô.

\subsubsection{Phân tích hàm lượng các chất có hoạt tính sinh học}

Qui trình ly trích mẫu: Cây thuốc dòi sau khi thu hoạch $\longrightarrow$ Rửa sạch loại bỏ tạp chất $\longrightarrow$ Phơi khô (để trong túi giấy phơi $3-5$ ngày, ẩm $\leq 12 \%$ ) $\longrightarrow$ Nghiền mịn (qua rây đường kính $3 \mathrm{~mm}$ ) $\longrightarrow$ Trích ly $\left(5 \mathrm{~g}\right.$ bột, $100 \mathrm{~mL}$ cồn $60 \%$ ở $60^{\circ} \mathrm{C}$ trong thời gian 60 phút, lặp lại 2 lần, trên bể điều nhiệt) $\longrightarrow$ Lọc qua giây lọc Whatman's No.1 $\longrightarrow$ Dịch trích $\longrightarrow$ Phân tích.

Phân tích anthocyanin theo phương pháp pH vi sai (Ahmed \& ctv., 2013; Santos \& ctv., 2013): Hút $2 \mathrm{~mL}$ các mẫu dịch trích nguyên chất cho vào các bình định mức $50 \mathrm{~mL}$, sau đó định mức đến vạch bằng dung dịch đệm $\mathrm{CH} 3 \mathrm{COONa} \mathrm{pH}$ $=4,5$ và dung dịch đệm $\mathrm{KCl} \mathrm{pH}=1,0$. Lắc dều và để ổn định trong 15 phút rồi tiến hành đo độ hấp thu của các mẫu ở bước sóng $520 \mathrm{~nm}$ và $700 \mathrm{~nm}$. Hàm lượng anthocyanin được tính toán theo công thức: Hàm lượng anthocyanin $(\mathrm{mg} / \mathrm{L})$ $=(\mathrm{A} \times \mathrm{MW} \times \mathrm{DF} \times 1000) /(\varepsilon \times 1)$. Trong đó, $\mathrm{A}=\left[\left(\mathrm{A}_{520 \mathrm{~nm}}-\mathrm{A}_{700 \mathrm{~nm}}\right) \mathrm{pH} 1,0\right]-\left[\left(\mathrm{A}_{520 \mathrm{~nm}}-\right.\right.$ $\mathrm{A}_{700 \mathrm{~nm}}$ ) pH 4,5]; MW (khối lượng phân tử của cyanidin-3-glycoside) $=449,2 \mathrm{~g} / \mathrm{mol} ; \mathrm{DF}$ (hệ số pha loãng); $\varepsilon=26900$ hệ số hòa tan của cyanidin3 -glycoside, lít $/ \mathrm{mol} / \mathrm{cm} ; 1=$ chiều dài của cuvet, $\mathrm{cm} ; 1000=$ hệ số chuyển đổi từ gram thành miligram.

Phân tích flavonoid theo phương pháp Aluminium Chloride Colorimetric (Eswari \& ctv., 2013; Mandal \& ctv., 2013). Hút 1 mL các mẫu dịch trích đã pha loãng/dung dịch chuẩn với nồng độ khác nhau cho vào ống nghiệm. Sau đó, cho tiếp $3 \mathrm{~mL}$ ethanol, $0,2 \mathrm{~mL}$ dung dịch $\mathrm{AlCl}_{3} 10 \%$, $2 \mathrm{~mL}$ dung dịch $\mathrm{CH}_{3} \mathrm{COONa} 1 \mathrm{M}$ và $5,8 \mathrm{~mL}$ nước cất. Các ống nghiệm được lắc đều với vortex. Giữ yên ở nhiệt độ phòng trong 30 phút. Đo độ hấp thu của hỗn hợp phản ứng ở 415 nm. Hàm lượng flavonoid được tính toán dựa vào đường chuẩn quercetin pha trong ethanol $(\mathrm{y}=$ $0,0054 \mathrm{x}+0,0026$ và $\left.\mathrm{r}^{2}=0,9995\right)$, kết quả thể hiện là miligram đương lượng quercetin trên gram nguyên liệu khô (mg QE/g DM).

Phân tích polyphenol theo phương pháp FolinCiocalteau (Hossain \& ctv., 2013). Hút 0,2 mL dịch trích đã pha loãng trong ethanol vào ống nghiệm và thêm $1,5 \mathrm{~mL}$ dung dịch thuốc thử Folin-Ciocalteu 10\%. Sau đó, các ống nghiệm được giữ trong tối 5 phút. Cuối cùng, cho thêm $1,5 \mathrm{~mL}$ dung dịch $\mathrm{Na}_{2} \mathrm{CO}_{3} 5 \%$ và lắc đều với vortex. Đem các ống nghiệm giữ trong tối 2 giờ. Sau đó, đo độ hấp thu ở bước sóng $750 \mathrm{~nm}$. Hàm lượng polyphenol được xác định dựa vào đường chuẩn của acid gallic pha trong ethanol $(\mathrm{y}=0,0082 \mathrm{x}$ $+0,0595$ and $\left.\mathrm{r}^{2}=0,9996\right)$, kết quả thể hiện là miligram đương lượng acid gallic trên gram nguyên liệu khô (mg GAE/g DM).

Phân tích tannin theo phương pháp FolinDenis (Laitonjam \& ctv., 2013). Hút $0,5 \mathrm{~mL}$ dịch trích đã pha loãng trong ethanol và $0,5 \mathrm{~mL}$ nước cất vào ống nghiệm, tiếp theo cho thêm 0,5 $\mathrm{mL}$ dung dịch thuốc Folin-Denis $10 \%$ và $2 \mathrm{~mL}$ $\mathrm{Na}_{2} \mathrm{CO}_{3} 20 \%$, lắc đều với vortex, làm nóng với nước sôi trong 1 phút. Sau đó để nguội xuống nhiệt độ phòng, đo độ hấp thu của phức màu ở bước sóng $700 \mathrm{~nm}$. Hàm lượng tannin được tính toán dựa vào đường chuẩn acid tannic pha trong ethanol $\left(\mathrm{y}=0,0098 \mathrm{x}+0,0478\right.$ và $\left.\mathrm{r}^{2}=0,9996\right)$, kết quả thể hiện là miligram đương lượng acid tannic trên gram nguyên liệu khô (mg TAE/g DM).

\subsubsection{Phân tích dữ liệu}

Số liệu thu thập được xử lý thống kê theo phần mềm EXCEL, SPSS 16.0. Dùng trắc nghiệm $\mathrm{F}$ (ANOVA) và phép thử DUNCAN để so sánh sự khác biệt giữa các nghiệm thức.

\section{Kết Quả và Thảo Luận}

\subsection{Thành phần dinh dưỡng trong đất thực nghiệm}

Để đánh giá độ chua của đất thì độ chua hiện tại $\mathrm{pH}$ nước là chỉ tiêu đánh giá khá quan trọng 
và rất cần thiết. Bảng 1 cho thấy $\mathrm{pH}$ dao động từ 5,8 - 6,0; còn ở mẫu đất trồng thuốc dòi tại xã Hòa Bình, huyện Chợ Mới là 5,6. Như vậy, $\mathrm{pH}$ nước của đất thích hợp cho cây thuốc dòi tím sinh trưởng và phát triển. Đất thí nghiệm có hàm lượng chất hữu cơ ở mức rất nghèo $(0,87 \% \mathrm{OM})$, nghèo hơn mẫu đất trồng thuốc dòi ở xã Hòa Bình $(3,21 \% \mathrm{OM}$, hàm lượng chất hữu cơ ở mức trung bình) (Do, 1999). Do đó, để tăng lượng chất hữu cơ cho đất, trong thí nghiệm có bón bổ sung thêm phân bò ủ hoai với số lượng 2 tấn/1000 m² .

Theo thang đánh giá của Truong (1994) đất thí nghiệm được đánh giá ở mức rất nghèo (đạm tổng số trung bình $0,05 \% \mathrm{~N}$ ), trong khi đó đất canh tác thuốc dòi tím ở xã Hòa Bình có đạm tổng số cao hơn $(0,14 \%$ N) (Bảng 1), do vậy cũng có thể ảnh hưởng đến năng suất cây thuốc dòi tím. Kết quả phân tích cũng cho thấy đất thí nghiệm kể cả đất canh tác thuốc dòi tím ở xã Hòa Bình có hàm lượng lân tổng số và $\mathrm{K}$ trao đổi ở mức rất thấp $(0,01 \mathrm{meq} / 100 \mathrm{~g}$ ) (Lê Văn Căn, trích dẫn bởi Ngo, 2005).

\section{2. Ảnh hưởng của mức độ bón phân đạm lên màu sắc lá của cây thuốc dòi}

\subsubsection{Chỉ số diệp lục tố (SPAD) trong lá}

Nitơ là nguyên tố tham gia vào thành phần cấu tạo nên diệp lục tố. Thiếu đạm diệp lục không hình thành và lá vàng tuy nhiên bón thừa đạm làm lá có màu xanh đậm, cây yếu và dễ bị sâu bệnh tấn công. Thông qua chỉ số SPAD ta có thể đánh giá hàm lượng diệp lục tố trong lá. Trên cây thuốc dòi nhận thấy sự hình thành diệp lục tố bị ảnh hưởng bởi lượng phân đạm bón. Bón mức đạm N4 (25 kg urea/1000 m²) nhận thấy lá xanh nhất và các mức độ đạm $\mathrm{N} 3, \mathrm{~N} 2$ và $\mathrm{N} 1$ màu xanh giảm dần và màu đỏ mặt trên lá tăng lên. Do đặc điểm cây thuốc dòi mặt trên lá màu lục, mặt dưới màu lục ánh tím đến tím; nên màu lục mặt trên giảm thì màu đỏ sẽ hiện rõ hơn (Hình 2).

Kết quả Bảng 2 cũng chứng minh mức độ bón phân đạm có ảnh hưởng rất lớn lên sự hình thành diệp lục tố trong lá cây thuốc dòi. Mức đạm N4 có chỉ số SPAD cao nhất là 40,68; sau đó lần lượt là $\mathrm{N} 3, \mathrm{~N} 2$ và thấp nhất là $\mathrm{N} 1$ với chỉ số $\mathrm{SPAD}$ là 31,41 ; sự khác biệt này có ý nghĩa thống kê ở mức ý nghĩa $(P \leq 0,01)$.

Màu tím đỏ trên thân và lá cây thuốc dòi bị ảnh hưởng bởi nhiều yếu tố như giống, lượng nước 
Bảng 2. Ảnh hưởng của mức độ bón phân đạm lên chỉ số SPAD và giá trị màu sắc a*

\begin{tabular}{cccc}
\hline Nghiệm thức & $\begin{array}{c}\text { Chỉ số diệp lục tố } \\
(\text { SPAD })^{1}\end{array}$ & $\begin{array}{c}\text { Giá trị a } \\
\text { lá mặt trên }\end{array}$ & $\begin{array}{c}\text { Giá trị a* } \\
\text { lá mặt dưới }\end{array}$ \\
\hline N1 $\left(10 \mathrm{~kg}\right.$ urea $\left./ 1000 \mathrm{~m}^{2}\right)$ & $31,41^{\mathrm{d}} \pm 0,52$ & $-5,76^{\mathrm{a}} \pm 0,15$ & $8,47^{\mathrm{b}} \pm 0,09$ \\
N2 $\left(15 \mathrm{~kg}\right.$ urea $\left./ 1000 \mathrm{~m}^{2}\right)$ & $34,92^{\mathrm{c}} \pm 0,43$ & $-5,74^{\mathrm{a}} \pm 0,11$ & $8,38^{\mathrm{b}} \pm 0,06$ \\
N3 $\left(20 \mathrm{~kg}\right.$ urea $\left./ 1000 \mathrm{~m}^{2}\right)$ & $38,36^{\mathrm{b}} \pm 0,85$ & $-6,18^{\mathrm{a}} \pm 0,08$ & $8,06^{\mathrm{b}} \pm 0,04$ \\
N4 $\left(25 \mathrm{~kg}\right.$ urea/1000 m$\left.{ }^{2}\right)$ & $40,68^{\mathrm{a}} \pm 0,91$ & $-6,78^{\mathrm{a}} \pm 0,12$ & $7,05^{\mathrm{a}} \pm 0,02$ \\
\hline Trung bình nghiệm thức & 36,34 & $-6,11$ & 7,99 \\
\hline F & $* *$ & $\mathrm{~ns}$ & $*$ \\
CV $(\%)$ & 15,18 & 17,56 & 6,90 \\
\hline
\end{tabular}

${ }^{1}$ Giá trị trung bình $(\mathrm{n}=10)$ và \pm độ lệch chuẩn $(\mathrm{SD}) ;{ }^{(\mathrm{ns})}$ Không khác biệt thống kê $(P>0,05)$; (**) Khác biệt thống kê ở mức $P \leq 0,01 ;\left(^{*}\right)$ Khác biệt thống kê ở mức $P \leq 0,05$.
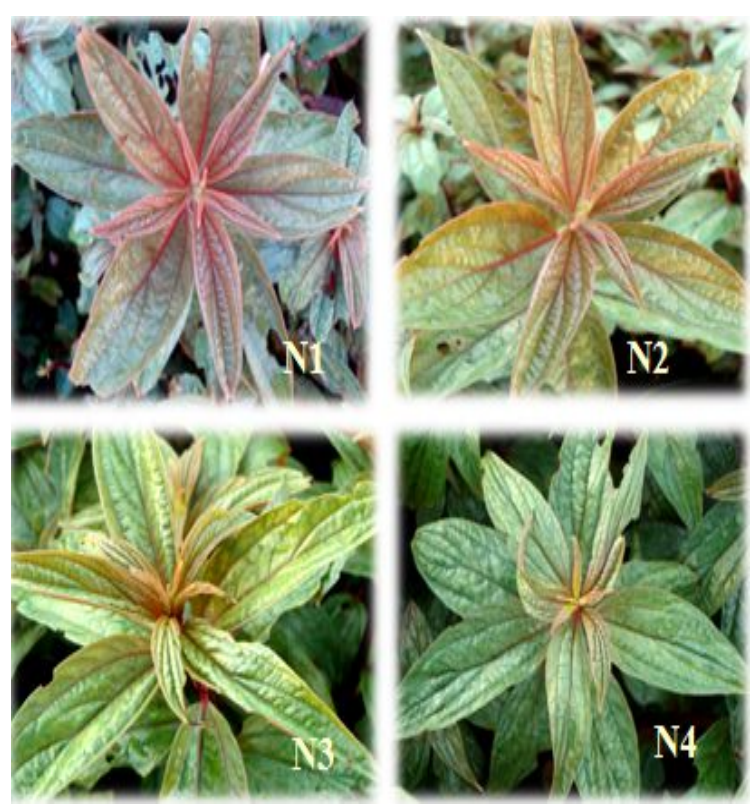

Hình 2. Ảnh hưởng của mức độ bón phân đạm lên sự hình thành diệp lục tố của lá.

tưới, ánh sáng, chế độ bón phân, mùa vụ,... Trong đó, mức độ bón phân đạm là yếu tố có ảnh hưởng lớn đến màu sắc lá. Để xác định chính xác sự thay đổi màu sắc lá thuốc dòi, nghiên cứu sử dụng máy đo màu (Chroma Meter CR - 400) để đo màu tím đỏ trên lá thuốc dòi. Trong đó chọn giá trị a* để theo dõi sự biến đổi về màu sắc của lá, giá trị $\mathrm{a}^{*}$ biến động trong khoảng từ -128 (xanh) đến 127 (đỏ), trị số $\mathrm{a}^{*}$ càng dương màu đỏ càng tăng dần.

Màu sắc lá được ghi nhận ở thời điểm thu hoạch của cây thuốc dòi và đo ở vị trí lá thứ ba và ở giữa lá. Kết quả ở Bảng 2 cho thấy trị số $\mathrm{a}^{*}$ của mặt trên lá chưa có sự khác biệt về thống kê $(P>$ $0,05)$. Tuy nhiên, ở mặt dưới lá thuốc dòi có màu tím đỏ và trị số a* đo được có sự khác biệt thống kê $(\mathrm{P} \leq 0,01)$, trong đó nhỏ nhất là mức đạm $\mathrm{N} 4$ $(7,05)$ và cao nhất là mức đạm $\mathrm{N} 1(8,47)$, giữa mức đạm $\mathrm{N} 1, \mathrm{~N} 2$ và $\mathrm{N} 3$ thì chưa có sự khác biệt về mặt thống kê. Kết quả cho thấy trong điều kiện của thí nghiệm với mức phân đạm bón 25 $\mathrm{kg}$ urea/1000 $\mathrm{m}^{2}$ làm cho màu tím đỏ trên thân lá cây thuốc dòi giảm đi (Hình 3).

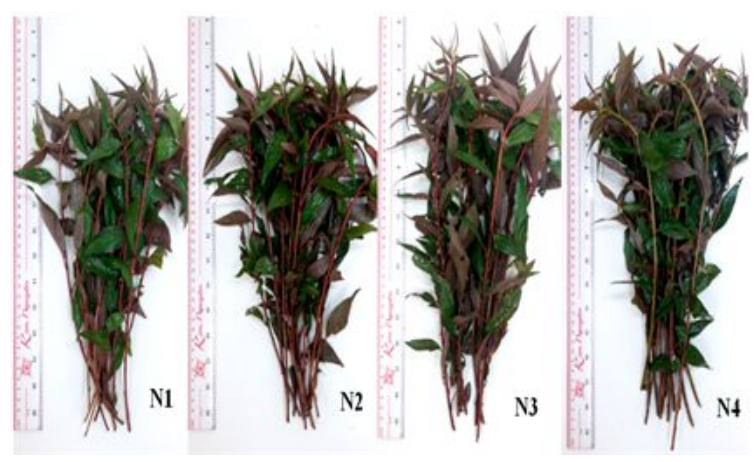

Hình 3. Ảnh hưởng của mức độ bón phân đạm lên màu sắc thân lá cây thuốc dòi.

Theo nghiên cứu của Yasemin \& ctv. (2017) cho thâyy nồng độ chlorophyll (thông qua giá trị SPAD) của lá cây oải hương tăng khi tăng nồng độ nitơ trong dung dịch tưới đến mức tối ưu và sẽ giảm xuống khi tiếp tục tăng nồng độ nitơ trong dung dịch tưới. Nồng độ chlorophyll được duy trì trong cây oải hương nhưng có giá trị thấp nhất khi nồng độ nitơ trong dung dịch tưới lên tới $800 \mathrm{mg} / \mathrm{L}$. Tương tự, Hassanpouraghdam \& ctv. (2008) cho rằng khi ứng dụng nitơ ở mức cao sẽ làm gia tăng cả hàm lượng chlorophyll của thực vật và tăng hiệu quả sử dụng nitơ trong sinh tồng hợp diệp lục tố. Họ phát hiện ra rằng hàm lượng chlorophyll (giá trị SPAD) của lá cúc tây tăng đáng kể khi tăng nồng độ nitơ trong dung dịch dinh dưỡng và hàm lượng nitơ lớn nhất được 
Bảng 3. Ảnh hưởng của mức độ bón phân đạm lên trọng lượng và năng suất cây thuốc dòi

\begin{tabular}{cccc}
\hline Nghiệm thức & $\begin{array}{c}\text { Trọng lượng tươi } \\
(\mathrm{g} / \mathrm{buui})\end{array}$ & $\begin{array}{c}\text { Trọng lượng khồ } \\
(\mathrm{g} / \mathrm{bụi})\end{array}$ & $\begin{array}{c}\text { Năng suất thực tế } \\
\text { tấn } / 1000 \mathrm{~m}^{2}\end{array}$ \\
\hline N1 $\left(10 \mathrm{~kg}\right.$ urea $\left./ 1000 \mathrm{~m}^{2}\right)$ & $53,13^{\mathrm{a}} \pm 0,62$ & $5,87 \mathrm{a} \pm 0,07$ & $1,32 \mathrm{a} \pm 0,06$ \\
N2 $\left(15 \mathrm{~kg}\right.$ urea $\left./ 1000 \mathrm{~m}^{2}\right)$ & $52,28^{\mathrm{a}} \pm 0,37$ & $6,32^{\mathrm{a}} \pm 0,05$ & $1,33^{\mathrm{a}} \pm 0,03$ \\
N3 $\left(20 \mathrm{~kg}\right.$ urea $\left./ 1000 \mathrm{~m}^{2}\right)$ & $81,72^{\mathrm{b}} \pm 0,89$ & $9,17^{\mathrm{b}} \pm 0,08$ & $1,64^{\mathrm{b}} \pm 0,05$ \\
N4 $\left(25 \mathrm{~kg}\right.$ urea $\left./ 1000 \mathrm{~m}^{2}\right)$ & $82,53^{\mathrm{b}} \pm 0,28$ & $8,12^{\mathrm{b}} \pm 0,06$ & $1,61^{\mathrm{b}} \pm 0,07$ \\
\hline Trung bình nghiệm thức & 67,42 & 7,37 & 1,48 \\
\hline F & $* *$ & $* *$ & $* *$ \\
CV $(\%)$ & 28,09 & 40,10 & 36,74 \\
\hline
\end{tabular}

${ }^{1}$ Giá trị trung bình $(\mathrm{n}=3)$ và \pm độ lệch chuẩn $(\mathrm{SD}) ;{ }^{(* *)}$ Khác biệt thống kê ở mức $P \leq 0,01$.

Bảng 4. Ảnh hưởng của các mức độ bón phân đạm lên hàm lượng các hợp chất sinh học trong cây thuốc dòi

\begin{tabular}{|c|c|c|c|c|}
\hline $\begin{array}{l}\text { Mức độ đạm } \\
\left(\mathrm{kg} / 1000 \mathrm{~m}^{2}\right)\end{array}$ & $\begin{array}{c}\text { Anthocyanin }^{1} \\
(\mathrm{mg} \mathrm{CE} / \mathrm{g} \mathrm{DM})\end{array}$ & $\begin{array}{c}\text { Flavonoid }^{1} \\
\left(\mathrm{mg} \mathrm{QE} / \mathrm{g} \mathrm{DM}^{2}\right)\end{array}$ & $\begin{array}{c}\text { Polyphenol }^{1} \\
(\mathrm{mg} \mathrm{GAE} / \mathrm{g} \mathrm{DM})\end{array}$ & $\begin{array}{c}\text { Tannin }^{1} \\
(\mathrm{mg} \text { TAE/g DM })\end{array}$ \\
\hline N1-10 & $0,285^{\mathrm{a}} \pm 0,003$ & $4,25^{\mathrm{b}} \pm 0,07$ & $11,94^{\mathrm{b}} \pm 0,08$ & $8,22^{\mathrm{b}} \pm 0,08$ \\
\hline $\mathrm{N} 2-15$ & $0,287^{\mathrm{a}} \pm 0,002$ & $4,37^{\mathrm{ab}} \pm 0,04$ & $11,87 \mathrm{~b} \pm 0,03$ & $7,83^{\mathrm{b}} \pm 0,45$ \\
\hline N3-20 & $0,296^{\mathrm{a}} \pm 0,005$ & $5,03^{\mathrm{a}} \pm 0,05$ & $15,54^{\mathrm{a}} \pm 0,14$ & $10,05^{\mathrm{a}} \pm 0,21$ \\
\hline N4-25 & $0,251^{\mathrm{b}} \pm 0,006$ & $3,36^{\mathrm{c}} \pm 0,06$ & $7,32^{\mathrm{c}} \pm 0,13$ & $5,68^{\mathrm{c}} \pm 0,15$ \\
\hline Trung bình & 0,280 & 4,25 & 11,67 & 7,95 \\
\hline $\mathrm{F}$ & $* *$ & ** & * & $* *$ \\
\hline $\mathrm{CV}(\%)$ & 17,67 & 20,97 & 20,07 & 7,56 \\
\hline
\end{tabular}
kê ở mức $P \leq 0,05$.

quan sát thấy là 400 mg/L.

\section{3. Ảnh hưởng của mức độ bón phân đạm đến trọng lượng và năng suất cây thuốc dòi khi thu hoach}

Phân đạm có vai trò sinh lý đặc biệt quan trọng đối với sinh trưởng phát triển và hình thành năng suất của cây trồng, giúp cây gia tăng nhanh chiều cao cũng như diện tích lá. Qua kết quả thí nghiệm cho thấy mức phân đạm có ảnh hưởng rất lớn đến sự gia tăng sinh khối của cây $(P \leq 0,01)$. Ở mức đạm bón $25 \mathrm{~kg} / 1000 \mathrm{~m}^{2}$ có trọng lượng tươi cao nhất $82,53 \mathrm{~g}$ /bụi, tuy nhiên chưa khác biệt có ý nghĩa với mức đạm bón $20 \mathrm{~kg} / 1000 \mathrm{~m}^{2}$. Trong khi đó, mức độ bón đạm $20 \mathrm{~kg} / 1000 \mathrm{~m}^{2}$ có trọng lượng khô và năng suất thực tế cao nhất lần lượt là $9,17 \mathrm{~g} /$ bụi và 1,64 tấn $/ 1000 \mathrm{~m}^{2}$ và chưa có sự khác biệt với mức đạm bón $25 \mathrm{~kg} / 1000 \mathrm{~m}^{2}$ (Bảng $3)$.

Theo kết quả nghiên cứu của Biesiada \& Kus (2010) trên rau húng quế cho thấy có sự gia tăng tổng năng suất trung bình từ $2,65 \mathrm{~kg}$ lên 4,05 $\mathrm{kg} / \mathrm{m}^{2}$ khi bón đạm tăng từ mức $50 \mathrm{~kg}$ lên 150 kg/ha. Khi tăng mức đạm cao hơn nữa 250 kg/ha thì năng suất tăng lên rất ít $4,34 \mathrm{~kg} / \mathrm{m}^{2}$. Sifola \& Barbieri (2006) đã chứng minh rằng, húng quế rất nhạy cảm với liều phân đạm bón, khi tăng dần liều phân đạm lên tới $300 \mathrm{~kg} / \mathrm{ha}$, trong điều kiện tưới nhỏ giọt, đã thu được sự gia tăng đáng kể năng suất theo trọng lượng tươi của các bộ phận trên mặt đất và lá lên tới 43,1 tấn/ha. Yassen \& ctv. (2003) đã báo cáo rằng sản lượng rau húng quế cao nhất (23,2 tấn/ha) khi bón phân đạm với liều tối ưu $120 \mathrm{~kg} / \mathrm{ha}$.

\section{4. Ảnh hưởng của mức độ bón phân đạm lên hàm lượng một số chất có hoạt tính sinh học có trong cây thuốc dòi}

Kết quả trình bày ở Bảng 4 cho thấy trong các mẫu cây thuốc dòi khô với các mức độ phân đạm bón khác nhau chứa hợp chất có hoạt tính sinh học khác nhau. Trong đó, nhiều nhất là polyphenol với hàm lượng từ $7,32-15,54 \mathrm{mg} / \mathrm{g}$ nguyên liệu khô $(\mathrm{DM})$; kế đến là tannin từ 5,68 -10,05 $\mathrm{mg} / \mathrm{g} \mathrm{DM}$; flavonoid từ $3,36-5,03 \mathrm{mg} / \mathrm{g} \mathrm{DM}$; và thấp nhất là anthocyanin từ $0,251-0,296 \mathrm{mg} / \mathrm{g}$ DM. Kết quả phân tích thống kê cho thấy có sự khác biệt rõ ràng giữa mẫu ở mức đạm N3 (20 kg 
urea/1000 m²) với các mẫu có mức đạm còn lại ở hàm lượng polyphenol và tannin, còn hàm lượng anthocyanin và flavonoid thì chưa có sự khác biệt thống kê giữa các mức đạm N1 - N3. Hàm lượng các hợp chất thu được cao nhất là ở mẫu N3; kế đến là $\mathrm{N} 1$ và $\mathrm{N} 2$; thấp nhất là $\mathrm{N} 4$. Như vậy, trong điều canh tác của thí nghiệm nếu bón với mức phân đạm cao $25 \mathrm{~kg}$ urea $/ 1000 \mathrm{~m}^{2}$ sẽ làm giảm hàm lượng những chất có hoạt tính sinh học trong cây thuốc dòi.

Hàm lượng phenol tổng và hoạt động chống oxy hóa trong quả ớt (Capsicum chinense) không bị ảnh hưởng bởi liều lượng phân đạm sử dụng, chỉ có hàm lượng acid tổng tăng lên gấp hai lần khi sử dụng phân đạm ở liều cao (Fidel \& ctv., 2011). Tuy nhiên, kết quả nghiên cứu của Bénard \& ctv. (2009) cho thấy khi bón phân đạm ở mức giới hạn có sự gia tăng hàm lượng polyphenol trong quả cà chua. Nghiên cứu của Uher \& ctv. (2013) thì cho thấy khi tăng hàm lượng nitrogen có sự gia tăng đáng kể các hợp chất sinh học như vitamin $\mathrm{C}, \mathrm{E}$ và beta carotene trong bông cải (Brassica oleracea var.); và nghiên cứu của Zhang \& ctv. (2016) trên cây cà chua cũng cho thấy hàm lượng acid cafeic trong quả tăng cao khi tăng lượng phân đạm còn hàm lượng rutin không khác biệt. Bên cạnh đó, kết quả nghiên cứu của Osuagwu \& Edeoga (2012) cho thấy khi tăng lượng phân bón vô cơ NPK có sự gia tăng đáng kể hàm lượng phenol và steroid trong lá cây hương nhu trắng (Ocimum gratissimum) và một loại cây rau gia vị ở Nigeria (Gongronema latifolium), không có sự ảnh hưởng khác biệt lên hàm lượng flavonoid trong lá của cây (Ocimum gratissimum) nhưng có sự gia tăng đáng kể flavonoid trong lá cây (Gongronema latifolium).

\section{Kết Luận}

Trong điều kiện thí nghiệm nhận thấy các mức độ bón phân đạm khác nhau có ảnh hưởng rõ rệt lên màu sắc lá, năng suất và hàm lượng các hợp chất sinh học của cây thuốc dòi. Kết quả nghiên cứu cho thấy với lượng bón $20 \mathrm{~kg}$ urea/1000 m² thì cây thuốc dòi khi thu hoạch có hàm lượng anthocyanin, flavonoid, polyphenol và tannin cao nhất, lần lượt là $0,296 \mathrm{mg} \mathrm{CE} / \mathrm{g}$ nguyên liệu khô (DM), 5,03 mg QE/g DM, 15,54 mg GAE/g DM và $10,05 \mathrm{mg} \mathrm{TAE} / \mathrm{g} \mathrm{DM}$; lá có màu tím đỏ với giá trị a* đo được là 8,06; chỉ số diệp lục tố SPAD là 38,36; năng suất 1,64 tấn/1000 $\mathrm{m}^{2}$. Tuy nhiên, với lượng phân đạm bón tăng lên $25 \mathrm{~kg}$ urea/1000 $\mathrm{m}^{2}$ thì hàm lượng các hợp chất có hoạt tính sinh học, màu tím đỏ của lá cây thuốc dòi và năng suất đều giảm.

\section{Tài Liệu Tham Khảo (References)}

Ahmed, J. K., Salih, H. A. M., \& Hadi, A. G. (2013). Anthocyanin in red beet juice act as scavenger for heavy metals ions such as lead and cadmium. International Journal of Science and Technology 2(3), 269-273.

Bénard. C. H., Gautier, F., Bourgaud, D., Grasselly, V., Navez, C., CarizVeyrat, M. W., \& Genard, M. (2009). Effects of low nitrogen supply on tomato (Solanum lycopersicum) fruit yield and quality with special emphasis on sugars, acids, ascorbate, carotenoids and phenolic compounds. Journal Agricultural and Food Chemistry 57(10), 4112-4123.

Biesiada, A., \& Kus, A. (2010). The effect of nitrogen fertilization and irrigation on yielding and nutritional status of sweet basil (Ocimum basilicum L.). Acta Scientiarum Polonorum, hortorum cultus 9(2), 3-12.

Bojović, B., \& Marković, A. (2009). Correlation between nitrogen and chlorophyll content in wheat (Triticum aestivum L.). Kragujevac Journal of Science 31, 6974 .

Chu, T. T., Pham, L. T., \& Nguyen, T. V. (2006). Medicinal plant cultivation techniques. Ha Noi, Vietnam: Labor and Social Publisher.

Do, R. T. T. (1999). A textbook of soil fertility and fertilizer. Can Tho, Vietnam: Can Tho University Publishing House.

Eswari, M. L., Bharathi, R. V., \& Jayshree, N. (2013). Preliminary phytochemical screening and heavy metal analysis of leaf extracts of Ziziphus oenoplia (L) Mill. Gard. International Journal of Pharmaceutical Sciences and Drug Research 5(1), 38-40.

Fidel, N. R., Daniel, G. M., Onésimo, G. J., \& Lourdes, C. D. (2011). Nitrogen fertilization effect on antioxidants compounds in fruits of habanero chili pepper ( Capsicum chinense). International Journal of Agriculture \& Biology 13(5), 827-830.

Hassanpouraghdam, M. B., Tabatabaie, S. J., Nazemiyeh, H., \& Aflatuni, A. (2008). N and K nutrition levels affect growth and essential oil content of costmary (Tanacetum balsamita L.). Journal of Food, Agriculture \& Environment 6(2), 145-149.

Hossain, M. A., Raqmi, K. A. S., Mijizy, Z. H., Weli, A. M., \& Riyami, Q. (2013). Study of total phenol, flavonoids contents and phytochemical sreening of various leaves crude extracts of locally grown Thymus vularis. Asian Pacific Journal of Tropical Biomedicine 3(9), 705-710.

Laitonjam, W. S., Yumnam, R., Asem, S. D., \& Wangkheirakpam, S. D. (2013). Evaluative and comparative study of biochemical, trace elements and antioxidant activity of Phlogacanthus pubinervius T. Anderson and Phlocanthus jenkincii C. B. Clarke leaves. Indian Journal of Natural Products and Resources $4(1), 67-72$. 
Le, T. T. (2007). Investigation of chemical composition of Avicennia. (Unpublished master' thesis). University of Science, Ho Chi Minh City, Vietnam.

Mandal, S., Patra, A., Samanta, A., Roy, S., Mandal, A., Mahapatra, T. D., Pradhan, S., Das, K., \& Nandi, D. K. (2013). Analysis of phytochemical profile of Terminalia arjuna bark extract with antioxidative and antimicrobial properties. Asian Pacific Journal of Tropical Biomedicine 3(12), 960-966.

Ngo, H. N. (2005). Reference scale for evaluation of some soil physical and chemical properties. College of Agriculture, Can Tho University, Can Tho, Vietnam.

Osuagwu, G. G. E., \& Edeoga, H. O. (2012). Effects of inorganic fertilizer application on the flavonoid, phenol and steroid content of the leaves of Ocimum gratissimum (L) and Gongronema latifolium (Benth). International Journal of Medicinal and Aromatic Plants 2(2), 254-262.

Paul, S., \& Saha, D. (2012). In vitro screening of cytotoxic activities of ethanolic extract of Pouzolzia Zeylanica (L.) Benn. International Journal of Pharmaceutical Innovations 2(1), 52-55.

Santos, D. T., Cavalcanti, R. N., Rostagno, M. A., Queiroga, C. L., Eberlin, M. N., \& Meireles, M. A. A. (2013). Extraction of polyphenols and anthocyanins from the Jambul (Syzygium cumini) fruit peels. Food and Public Health 3(1), 12-20.

Sifola, M. I., \& Barbieri, G. (2006). Growth, yield, and essential oil content of three cultivars of basil grown under different levels of nitrogen in the field. Scientia Horticulturae 108(4), 408-413.
Truong, N. T. (1994). A textbook of soil fertility. Can Tho, Vietnam: Can Tho University Publishing House.

Uher, A., Slosar, M., \& Valsikova, M. (2013). Fertilisation impact on the content of selected bioactive compounds in Cauliflower. Journal of Central European Agriculture 14(1), 261-269.

Vo, C. V. (2012). Dictionary of Vietnamese medicinal plants. Ha Noi, Vietnam: Medical Publishing House.

WHO (World Health Organization). (2003). WHO guidelines on good agricultural and collection practices for medicinal plants. Retrived September 1, 2018, from https://lhu.edu.vn/Data/News/602/files/3_GACPW HO_Vietnamese_2dkpy.doc.

Yasemin, S., Ozkaya, A., Koksal, E., Gok, B. (2017). The effects of nitrogen on growth and physiological features of lavender. International Congress on Medicinal and Aromatic Plants 10-12, 746-754.

Yassen, M., Ram, P., Yadav, A., \& Singh, K. (2003). Response of Indian basil (Ocimum basilicum) to irrigation and nitrogen schedule in Central Uttar Pradesh. Annals of Plant Physiology 17(2), 177-181.

Zhang, E., Duan, Y., Tan, F., \& Zhang, S. (2016). Effects of long-term nitrogen and organic fertilization on antioxidants content of tomato fruits. Journal of Horticulture 3(1), 1-5. 